Session 3548

\title{
A Simple Software and Hardware System Solution for Process Measurement and Control in Engineering Technology Student Design Projects
}

\author{
Dale H. Litwhiler \\ Penn State Berks-LehighValley College
}

\begin{abstract}
Many design projects conceived by engineering technology students involve using a computer to control some form of physical process. These hardware-in-the-loop (HIL) projects involve making measurements of some physical quantities, processing the measured data, and producing control signals based on the processed data. Choosing the appropriate measurement and control system to achieve the design objectives is often a difficult part of the design. Most projects require the measurement and control of very few parameters that does not justify the purchase of an elaborate analog and digital I/O system. Combining LabVIEW ${ }^{\mathrm{TM}}$ software with the 1-Wire ${ }^{\mathrm{TM}}$ family of integrated circuit devices from Dallas-Maxim produces a measurement and control system that is an excellent match for many student design projects. LabVIEW is very popular in engineering technology programs and provides the software tools needed to easily develop the data acquisition, signal processing and control routines. The student version of LabVIEW $6 \mathrm{i}$ provides plenty of computing power for such applications, is very affordable, and runs on either Mac or IBM-compatible computers. For the measurement and control hardware, 1-Wire devices provide a flexible and low cost set of analog and digital input and output devices that can communicate with the computer via the serial port. Devices in the 1-Wire family include temperature sensors, A/D converters, counters, digital I/O and digital potentiometers. The 1Wire device bus scheme allows for many devices to be connected to the computer simultaneously, addressed individually, and powered directly from the bus. This paper presents and discusses the software and hardware necessary to implement HIL student design projects on both Mac and IBM-compatible computers. Examples of the LabVIEW code and sensor and actuator designs are also presented.
\end{abstract}

Introduction

Design projects are an excellent tool for motivating engineering technology students. Although design is not typically emphasized in engineering technology programs, the design project exercise provides an opportunity for the students to apply scientific and mathematical knowledge to convey an engineering experience. ${ }^{1}$ Many student design projects involve measuring a physical quantity and performing a control function based on this measurement. The design project does not always involve starting from scratch but rather pulling together a collection of existing components or subsystems into a system to achieve the design objective. Perhaps the 
student has an existing circuit, device, or system that lends itself to being integrated into an automated system. Here the project entails interfacing the real-world with a computer which adds another level of sophistication to the design but also introduces new technical hurdles to get over: data acquisition and control software and hardware. ${ }^{5}$ Projects like this are encouraged as they represent the prominent and growing system design philosophy of embedded processors and hardware-in-the-loop (HIL) control systems. These systems are very flexible and can be upgraded and modified by simply changing the software (or firmware). Planning ahead for inevitable system change is an important lesson for engineering technology students.

LabVIEW software is popular in engineering technology curriculum and students are often familiar with at least using LabVIEW applications. The ease of use, low cost and functionality of the student version of LabVIEW helps to make it a natural first choice as the software for student design projects. ${ }^{2}$ Even students that are not familiar with structured programming can quickly be creating LabVIEW code. ${ }^{3}$ The student version also runs on either IBM-compatible (PC) or Apple Macintosh (Mac) computers which makes it ideal for home use by the students. This capability allows the students to work on the software on there own schedule without relying on laboratory access.

The choice of the computer interface hardware is more challenging due to the plethora of data acquisition and control devices, boards and systems available on the market. Available computer interface components include those using the IEEE-488 bus, PCI/ISA plug-in cards, VXI/PXI cards, PCMCIA cards, USB, parallel port, and RS-232 (serial port). Each of these interfaces has its particular application-specific benefits. However, most student projects require the measurement and control of very few parameters that does not justify the purchase of an elaborate analog and digital I/O system. In these situations, the Dallas-Maxim 1-Wire devices provide an elegant solution.

The 1-Wire integrated circuit devices produced by Dallas-Maxim are connected to the controlling processor via one conductor and a ground connection (hence the name, "1-Wire"). Both device power and the bi-directional communication signals are sent through the same single conductor. Each device has a unique factory-set identification address therefore many devices can be connected to the 1-Wire bus simultaneously to form what is called a MicroLAN. ${ }^{4}$ Devices in the 1-Wire family include temperature sensors, A/D converters, counters, digital I/O and digital potentiometers. These devices provide an excellent set of building blocks to be embedded into student projects. Many of these devices are readily available through distributors such as Newark and Digi-key but students are encouraged to obtain devices as free samples directly from the manufacturer. ${ }^{6} \quad 1$-Wire devices are designed to be connected directly to a microcontroller I/O pin or to a host computer via a simple inexpensive serial port adapter. The 1-Wire devices are easy to connect to form the MicroLAN which allows the students to spend less time debugging hardware and more time developing creative software for their project.

Another key benefit of using inexpensive (or free) 1-Wire devices is that the finished project remains the property of the students and does not have to be dismantled to return equipment to the school. This is very important in motivating the students to come up with useful and practical design projects. The students design the system with long-term personal usage in mind. 


\section{1-Wire MicroLAN Overview}

Figure 1 shows a schematic layout of a typical 1-Wire system connected to a PC. All communication between the computer and the 1-Wire devices is through the DS9097U adapter connected to the serial port. Power for the adapter electronics is derived from the Data Terminal Ready (DTR) and the Request To Send (RTS) signals of the RS-232 serial port. Communication between the PC and the DS9097U adapter is done with simple hexadecimal strings written to and read from the serial port. System wiring can be done with simple telephone wire for short runs $\left(<100 \mathrm{ft}\right.$ ) or category 5 (CAT5) data communication wire for runs up to $300 \mathrm{~m}^{7}$

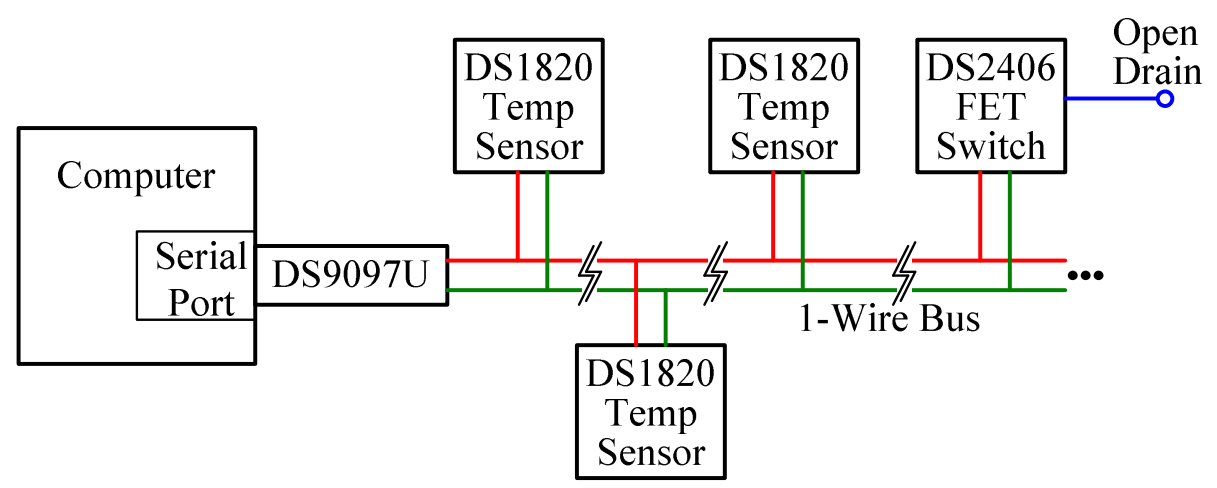

Figure 1. Typical MicroLAN Layout

Devices can be connected (dropped) anywhere along the line. This architecture greatly simplifies system wiring. Clusters of devices can be created as needed and can be a great distance from the host. Each device contains a unique 64 bit address that is programmed at the factory. Therefore any combination of devices can be connected and each device uniquely addressed and controlled. Within each device, a half-wave rectifier and hold-up capacitor circuit is used to derive chip power from the signal line. Data transmitted by each 1-Wire device is accompanied by a cyclic redundancy check (CRC) to allow the host to check for data transmission errors. Devices are rated for operation over a wide temperature range of $-40^{\circ} \mathrm{C}$ to $+85^{\circ} \mathrm{C}$ making them well suited to outdoor sensing and control projects. ${ }^{6}$

Figure 2 shows a photograph of the DS9097U adapter and a few devices representing the available packages as indicated on the figure. The DS1820 temperature sensor, DS2890 digital potentiometer, and DS2406 switch are available in the 3-leaded TO-92 package. Most other devices are only available in the TSOC or SOIC package which makes soldering a bit challenging.

Figure 3 shows a few homebrew methods of connecting to the devices. A DS1820 (TO-92 package) temperature sensor is shown sealed inside a piece of shrink tubing with silicone RTV. This type of packaging is suitable for outdoor or liquid temperature measurement. Also shown in Figure 3 are two SOIC packages soldered onto a homemade printed circuit board to allow easier wire connections. Another connection technique shown in Figure 3 is a TSOC package with small strands of wire soldered from its leads to the prongs of a standard component carrier. 
The carrier can then be plugged into a socket or a prototype board. Various adapter and converter printed circuit boards are also available commercially.

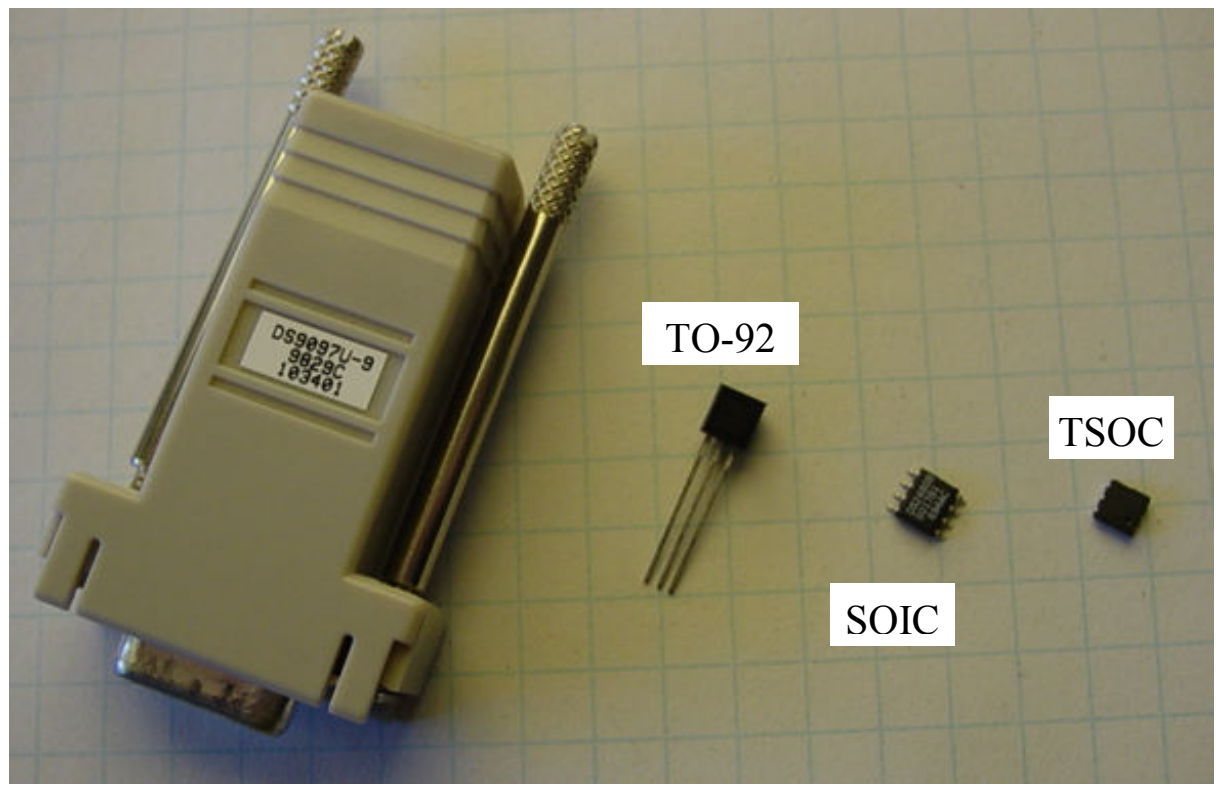

Figure 2. DS9097U Adapter and Device Packages

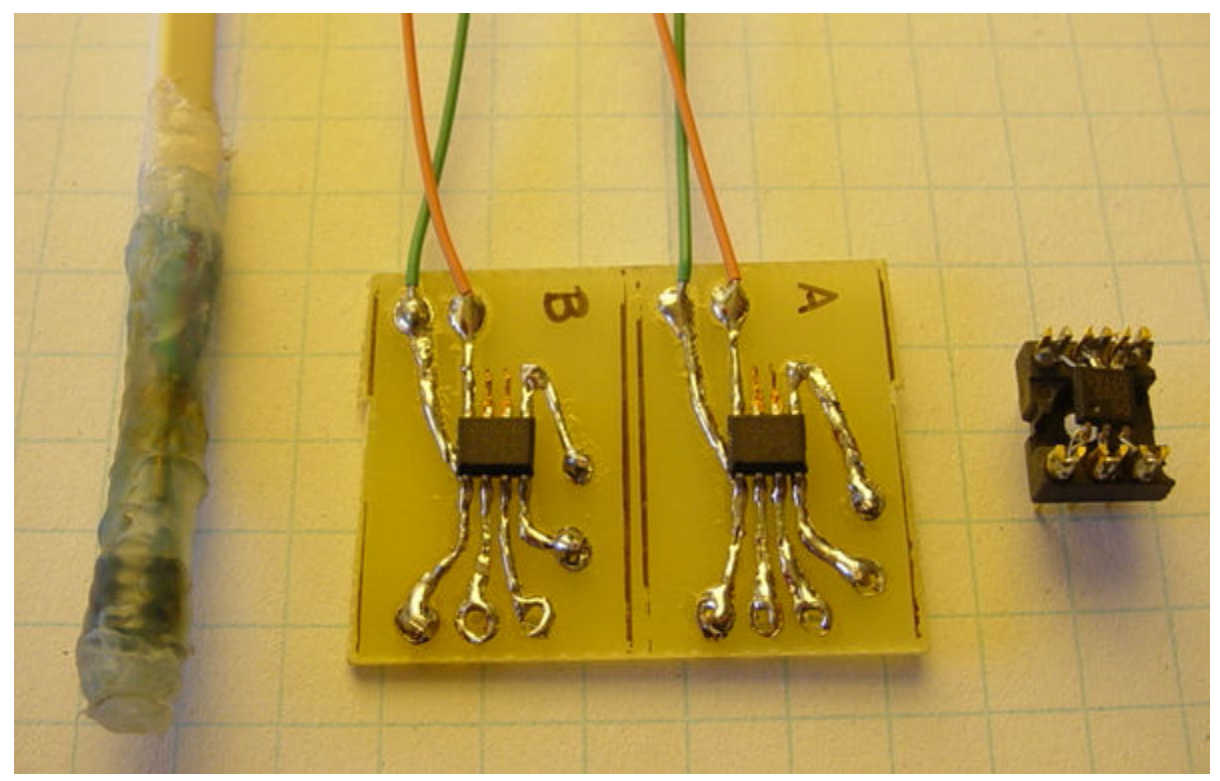

Figure 3. Device Connection Examples 


\section{LabVIEW Code Overview}

The string and format conversion built-in functions of LabVIEW are well suited to communicating with the DS9097U adapter. By writing and reading single-byte hexadecimal strings to and from the PC serial port, any 1-Wire device can be addressed and controlled. The DS9097U appears to the PC like any other serial device and is treated as such in the software. Command bytes are written to the desired COM port and response bytes are read from the COM port buffer. Each device datasheet contains a complete list of commands as well as example code that must then be translated into LabVIEW "G" code. Device-level LabVIEW Virtual Instruments (VIs) have been developed by the faculty and made available to interested students to reduce project development time and to keep the students focused on the main parts of the design. These VIs were written entirely within LabVIEW with no external libraries, drivers or code required. By doing this, the MicroLAN can be connected to either PC or Mac computers. ${ }^{8}$

The device-level VIs (or subVIs), help to make the students' LabVIEW diagram designs more intuitive and frees them from the task of writing arrays of hexadecimal commands to each device. Rather, each subVI essentially represents a function to be performed. For the temperature sensors, the function is to return the temperature measured by the addressed device. For the FET switch, one subVI was developed to simply turn it on or off while another subVI was designed to read back the status of the switch. Using this functional approach allows the students to build their LabVIEW diagrams in a functional manner much like their conceptual "back of the envelop" system design.

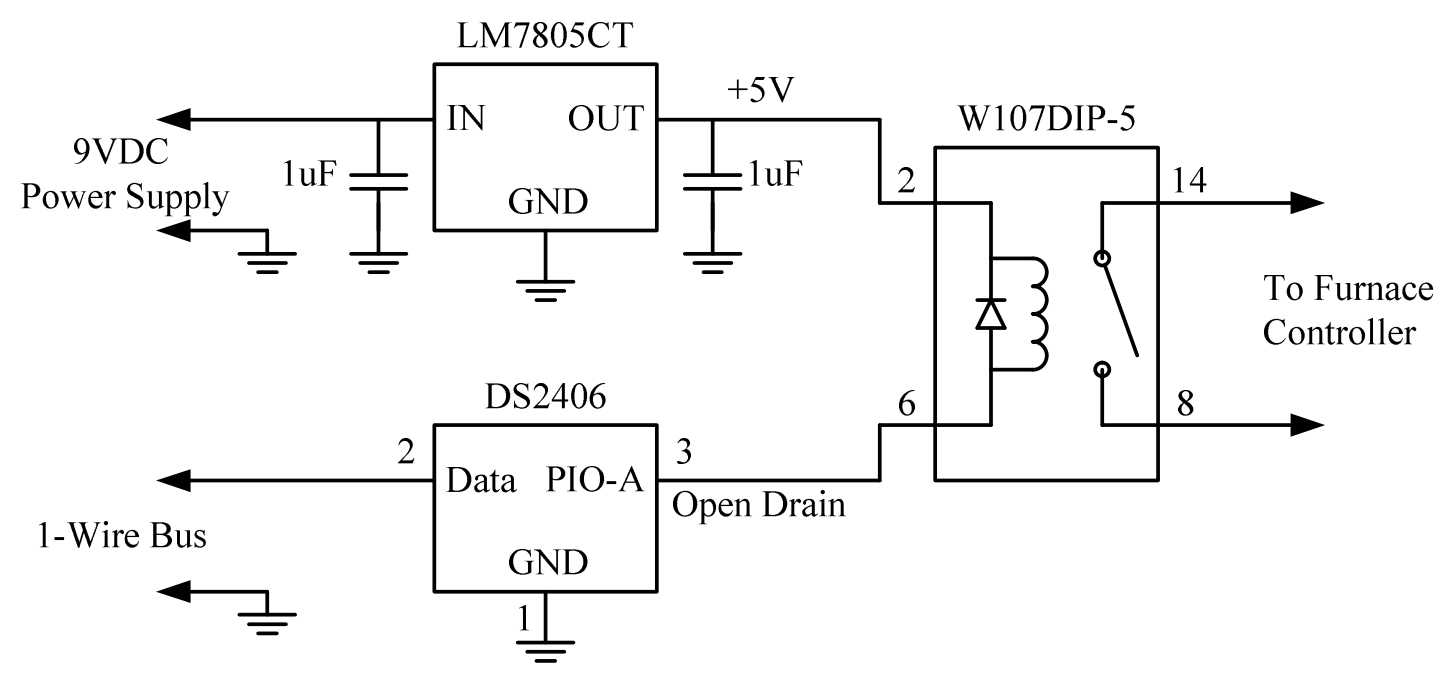

Figure 4. Schematic of 1-Wire Furnace Controller Interface Board

\section{Example Project}

A group of senior electromechanical engineering technology students chose to design a computer-controlled home HVAC system as their senior design project. The students first sketched out the basic operation of the system. The MicroLAN layout in Figure 1 shows the 
conceptual design of the heating portion of this process control system. The temperature sensors are located in various rooms of the house. The FET switch is located at the furnace location where its open drain output is used to energize a relay to turn on the furnace. Figure 4 shows the schematic of the furnace controller interface board. With 1-Wire devices, the hardware design is quite simple. The software contains the system personality and is used to close the loop on the process control.

Figure 5 shows an example front panel (user interface) of the LabVIEW VI in which room temperatures are measured and averaged to control the ON-OFF cycling of a forced hot air furnace. The LabVIEW front panel displays the temperature reading for each of the rooms in graphical and numeric formats. Also displayed is the current status of the furnace. The user selects the temperature set point and the amount of temperature hysteresis (swing). Engineering technology students are very computer savvy and the design of the front panel is their opportunity to make a user interface just the way they want it.

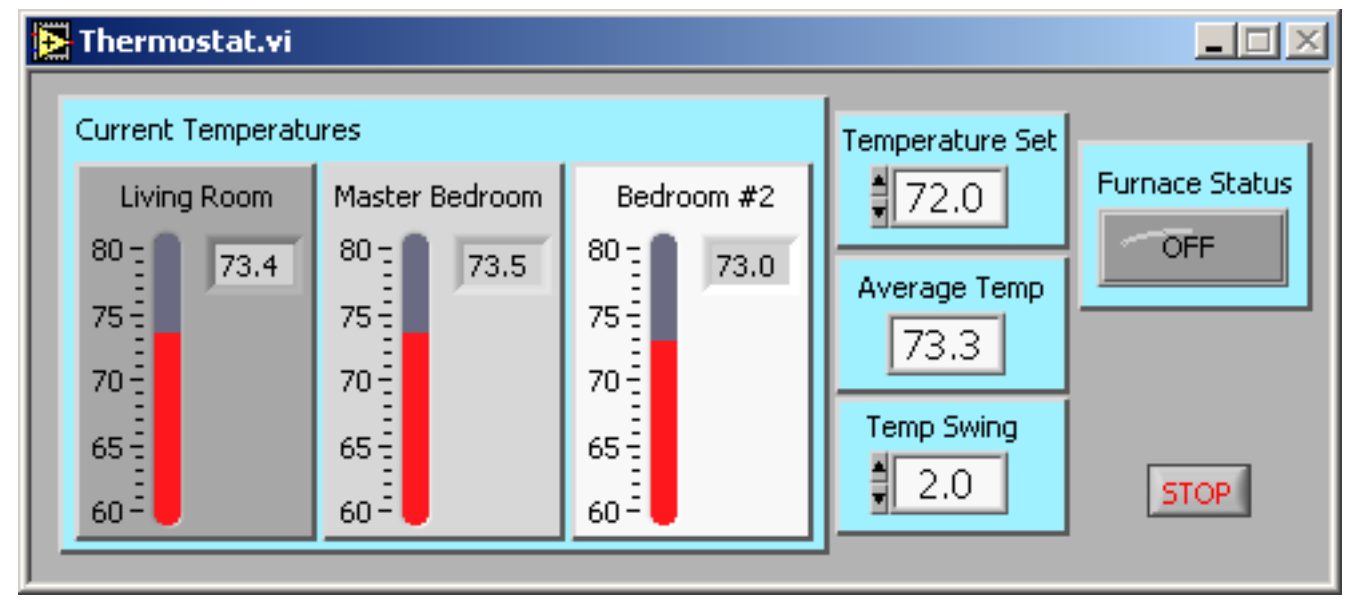

Figure 5. LabVIEW Example Front Panel User Interface

The LabVIEW diagram of Figure 6 shows the "G" code implementation of the heating process control algorithm. The 1-Wire device communication subVIs, temperature averaging mathematics and temperature threshold comparison is shown. Here is where a large part of the actual system design takes place. Implementing the sensing and control algorithm is the heart of the project. The 1-Wire device subVIs greatly streamline the software design. The students can design the software much like they would sketch out a block diagram of the system function. If a temperature measurement is needed, the DS1820 subVI is placed in that location on the diagram and wired to the MicroLAN address of the appropriate device. Similarly, the DS2406 FET switch subVI is placed where needed with a simple Boolean constant setting whether the switch is commanded on or off. 


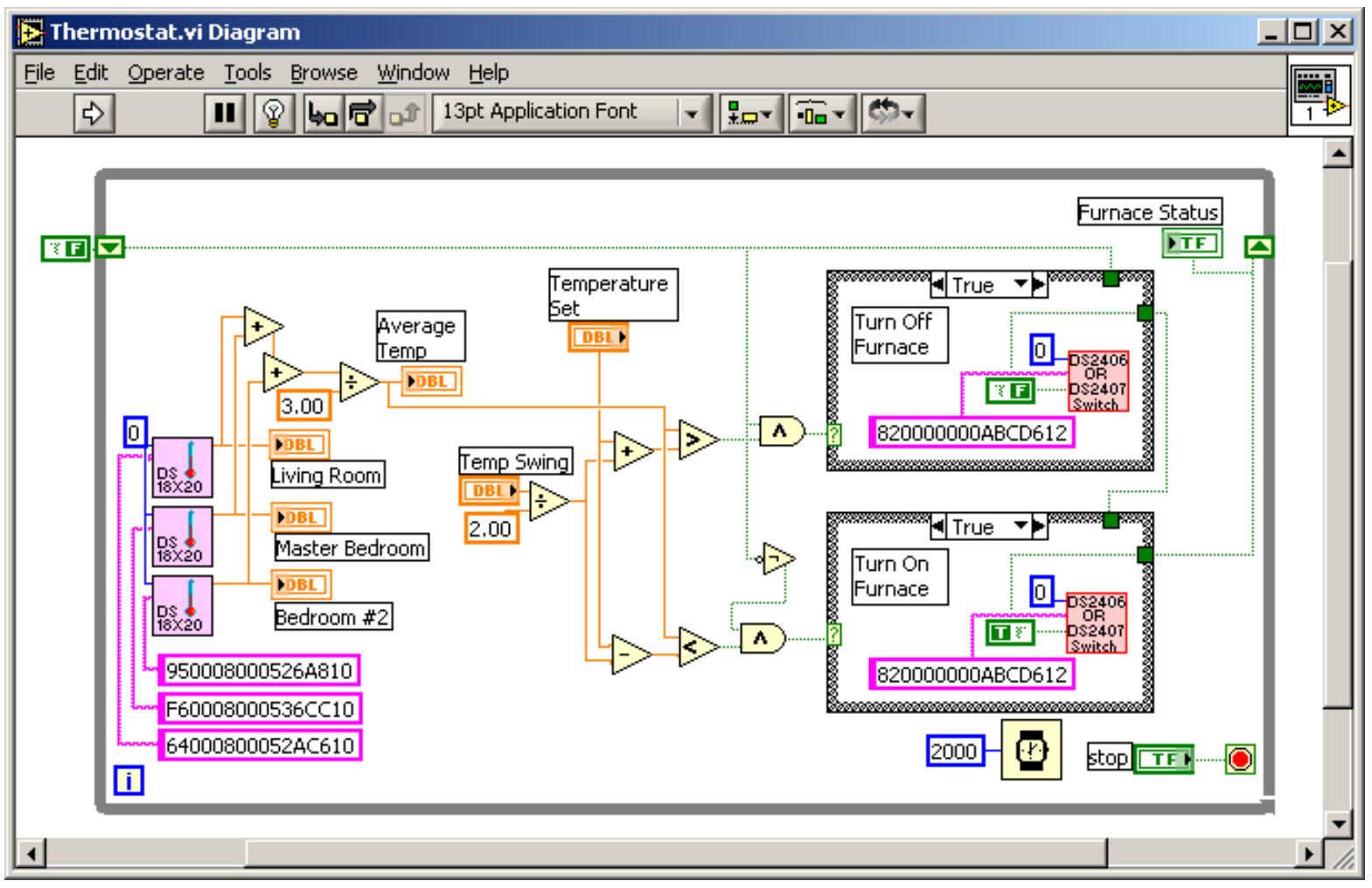

Figure 6. LabVIEW Example Diagram

On the hardware side of the project, the students appreciated the ease with which the MicroLAN could be wired into a home heating system. The multi-drop architecture of the MicroLAN also allows for easy system expansion to include other sensing and control elements. System expansion might include sensing relative humidity in the rooms and controlling a humidifier water valve at the furnace. ${ }^{9}$ If the heating system is equipped with dampers to control the flow of heated air to different zones of the house, control for these dampers can easily be added to the software and hardware. Here again the concept to be stressed to the students is the planning ahead for unforeseen but inevitable changes by specifying upgradeable hardware and software.

\section{Conclusions}

In student design projects involving process control, the combination of LabVIEW and 1-Wire devices provides an elegant solution. The architecture of such a system allows the students to focus on the overall system design rather than getting lost in the details of computer interface hardware design. The functions available in the 1-Wire family of devices create a very attractive palette for process control applications.

The software and the hardware presented here demonstrate the important ability to be changed and upgraded easily. With this system, students are more likely to succeed in producing a functioning project which therefore helps to provide a more motivating and confidence building experience. Engineering technology students quickly recognize the power of the software for 
making changes and improvements to the design of the overall system without changing any of the hardware design. With a computer running LabVIEW in the control loop, the application can be made very elaborate. Energy usage reports can be generated. Sound files can be played to provide audible warnings or indicators of certain sensed conditions. If the controlling computer is connected to the Internet, system status and data can even be published on a web page. This type of capability is very attractive to modern engineering technology students.

\section{Bibliography}

1. Lopez, G. W. and Hansberry, E. W., "Mini-design projects; a Hands-on Approach to Teaching Instrumentation Courses in ET Programs," Proceedings of the American Society for Engineering Education Annual Conference and Exposition, 2003.

2. Rogers, C., "Data Acquisition for the Dorm Room: Teaching Experimentation Techniques Using LEGO Materials," Proceedings of the American Society for Engineering Education Annual Conference and Exposition, 2001.

3. McClain, S. T. and Cain, B., "Teaching Modern Data Acquisition Systems with a Departmental Requirement for Student Laptop Ownership," Proceedings of the American Society for Engineering Education Annual Conference and Exposition, 2003.

4. Awtrey, D., “Transmitting Data and Power over a One-Wire Bus,” February 1997, Sensors, Vol. 14, No. 2.

5. Braun, C. G., "Experiments on the Cheap: Using a Student Data Acquisition System," Proceedings of the American Society for Engineering Education Annual Conference and Exposition, 1997.

6. $\quad$ http://www.maxim-ic.com

7. $\quad$ http://www.dalsemi.com/TechBriefs/tb1.htm

8. Litwhiler, D.H., "A Versatile LabVIEW Environment for Communicating with Dallas/Maxim1-Wire Devices," submitted for publication, ASEE Computers in Education Journal.

9. Awtrey, D., “A 1-Wire Humidity Sensor,” August 2000, Sensors, Vol. 17, No. 8.

Biography

\section{DALE H. LITWHILER}

Dale H. Litwhiler is an Assistant Professor at Penn State Berks-Lehigh Valley College in Reading, PA. He received his B.S. from Penn State University (1984), his M.S. from Syracuse University (1989) and his Ph.D. from Lehigh University (2000) all in electrical engineering. Prior to beginning his academic career in 2002, he worked with IBM Federal Systems and Lockheed Martin Commercial Space Systems as a hardware and software design engineer. 